\title{
A Comparative Study of Unenhanced Focused Appendiceal CT and Sonography for the Diagnosis of Acute Appendicitis
}

\author{
Prabhakaran A V
}

Associate professor, Department of Radiology, IRT Perundurai Medical College and Hospital, Perundurai, Tamil Nadu, INDIA. Email: cameoprabhakar@yahoo.com

\begin{abstract}
Background: The newer techniques of ultrasonography (US) and computed tomography (CT) have shown great promise in evaluation of patients with suspected acute appendicitis. Focused appendix CT technique is designed to minimize the time, radiation exposure, and patient discomfort in patients with suspected appendicitis. Aim: To compare the diagnostic performances of Ultrasound (graded compression Sonography) and unenhanced focused CT in patients suspected of having acute appendicitis. Material and Methods: In this prospective study, an analysis of 61 consecutive patients with acute appendicitis was done. CT examination was performed with four slice helical CT scanner (Asteion, Toshiba) by means of a rapid thin-scanning technique. Ultrasonography was performed using Nemio Toshiba machine using a 4.2-MHz convexarray transducer. Results: CT had a sensitivity of $100 \%$, specificity of $96.97 \%$ and accuracy of $98.36 \%$ for the diagnosis of acute appendicitis. There was discordance between CT and USG in the diagnosis of appendicitis in 6 cases. CT diagnosis was correct in all 6 cases ( 2 false positive in USG were correctly reported as no appendicitis by CT, and 4 cases missed by USG were correctly diagnosed as appendicitis by CT). Conclusion: This study shows that both unenhanced focused CT and sonography are accurate imaging modalities in patients with suspected appendicitis. However, CT was found to be superior to US in evaluating patients suspected of having acute appendicitis.

Key Word: Acute appendicitis, focused appendix CT technique, graded compression sonography, sensitivity, specificity
\end{abstract}

*Address for Correspondence:

Prabhakaran. A V, Department of Radiology, IRT Perundurai Medical College and Hospital, Perundurai, Tamil Nadu, INDIA.

Email: cameoprabhakar@yahoo.com

Received Date: 10/11/2019 Received Date: 12/12/2019 Accepted Date: 26/01/2020

DOI: https://doi.org/10.26611/10131321

\begin{tabular}{|l|l|}
\hline \multicolumn{2}{|c|}{ Access this article online } \\
\hline Quick Response Code: & \multirow{2}{*}{ Website: } \\
\hline www.medpulse.in \\
\hline
\end{tabular}

\section{INTRODUCTION}

Acute appendicitis, the most common abdominal surgical emergency can be diagnosed clinically with great confidence in most cases. Some patients may present with atypical clinical features and non-specific physical findings, which preclude early diagnosis and leads to complications. Moreover, the rate of negative findings for appendicitis at laparotomy or laparoscopy based on clinical features may be as high as $50 \% .^{1-3}$ The newer techniques of ultrasonography (US) and computed tomography (CT) have shown great promise in evaluation of patients with suspected acute appendicitis. US is a simple, rapid, noninvasive and inexpensive modality which is not associated with ionising radiation. It can be used as a screening modality for initial evaluation of such patients. But US is highly operator-dependent, and excessive bowel gas hinders proper evaluation of the appendix. Further, reports suggest that the sensitivity of US is $76 \%-90 \%$, specificity is $86 \%-100 \%$. In comparison, CT is readily available, relatively easy to perform, and has results that are easy to interpret. Unenhanced helical CT provides global crosssectional evaluation and important information regarding the appendix, mesentery and retroperitoneum. A distinct advantage of unenhanced helical $\mathrm{CT}$ is the short examination time because it does not require patient preparation or contrast administration. Further, reports underscores that helical CT has sensitivities of $70-100 \%$ and specificities of $91-99 \%$. Recently, focused appendix 
CT is a technique that employs contiguous, thin collimation helical scanning limited to the right lower quadrant. This technique is designed to minimize the time, radiation exposure, and patient discomfort in patients with suspected appendicitis. The present study was carried out to compare the diagnostic performances of Ultrasound (graded compression Sonography) and unenhanced focused CT in patients suspected of having acute appendicitis.

\section{MATERIAL AND METHODS}

In this prospective study, an analysis of 61 consecutive patients who presented to our hospital with acute appendicitis was done.

\section{Inclusion criteria}

- Patients with clinical suspicion of appendicitis.

Exclusion criteria

- Pregnant patients

- patients with previous history of appendicectomy

- unwilling patients

\section{CT Examination}

CT examination was performed with four slice helical CT scanner (Asteion, Toshiba) by means of a rapid thinscanning technique. A single breath hold helical scan from the top of the L2 vertebral body to the pubic symphysis was obtained in supine position. The technical parameters were as follows: collimation of $4 \times 5 \mathrm{~mm}$, table speed of $17.5 \mathrm{~mm}$ per rotation, pitch of 0.825 , rotation time of 0.75 seconds, $120 \mathrm{kVp}$, and $100-240 \mathrm{~mA}$. The axial section data were reconstructed with a $5-\mathrm{mm}$ thickness at $5-\mathrm{mm}$ intervals and viewed using different soft-tissue window settings (width, $450 \mathrm{HU}$; level, $50 \mathrm{HU}$ ). No oral, rectal, or IV contrast material was administered. CT scan images were analyzed both at a workstation and on hard copy.

The following observations were made on CT examinations:

- Whether appendix was visible, if seen its maximal outer transverse diameter, and

- The presence or absence of following findings:(a) gas in the appendiceal lumen, (b) Appendicolith, (c) periappendiceal fat stranding, (d) caecal wall thickening, and (e) abscess or phlegmon in the right iliac fossa. Each finding was separately coded.

- If the above findings were absent, a general survey of visualized parts of abdomen to find an alternative diagnosis that could explain the patient's symptoms was done.

CT findings were diagnostic of appendicitis if the outer transverse diameter of appendix is $\geq 6 \mathrm{~mm}$ with or without appendicolith. If the appendix was not visualised then the presence of abscess or phlegmon is taken as a positive criteria. The presence of gas in the appendiceal lumen was considered as a possible negative criterion for acute appendicitis.

\section{US Examination}

Ultrasonography was performed using Nemio Toshiba machine. A general survey of entire abdomen, including the pelvis, was performed in all patients by using a 4.2$\mathrm{MHz}$ convex-array transducer. In all the patients a targeted scan of right lower quadrant was done after emptying the bladder, with a 6-9 MHz linear-array transducer with use of the graded compression technique described by Puylaert 8. Before the study the patients was asked to point to the site of maximal pain in the right lower quadrant with a single finger. The examination was initiated by scanning in the transverse plane in the right lateral and mid abdomen just above the level of umbilicus. The examination was continued caudally to the right lower quadrant with gradually increasing compression. Compression was increased until all bowel gas and fluid could be expressed from the ascending colon and caecum. The normal caecum could be compressed by moderate pressure. Care was taken so that transducer pressure was gradually increased. The inflamed appendix was most often visualised at the base of the caecum during maximal graded compression as a tubular blind ending non-peristaltic structure with bowel signature. The examination was continued caudally with identification of psoas and iliacus muscles, and the external iliac vessels. Longitudinal and oblique scans were also obtained, again with graded compression.

The following observations were made on US examinations:

- Whether appendix was visible, if seen its maximal outer transverse diameter

- Presence or absence of following findings : (a) fluid-filled appendix, (b) lack ofcompressibility of the appendix, (c) appendicolith, (d) pericecal fluid, (e) hyperechoicperiappendiceal tissue, (f) abscess or phlegmon, and (g) maximal tenderness at the site of the appendix.

- If the above findings were absent, a general survey of abdomen to find an alternative diagnosis that could explain the patient's symptoms was done.

The appendiceal diameter of $\geq 6 \mathrm{~mm}$ in a noncompressible appendix, with or without appendicolith was considered as positive criteria. If the appendix is not visualised then the presence of abscess or phlegmon was taken as a positive criteria.

Final diagnosis

The CT and US findings was independently reported as (a) suggestive of appendicitis, (b) no evidence of appendicitis, or (c) An alternative diagnosis 
Definite diagnosis

In all patients who underwent surgery, definite diagnosis was made on the basis of operative findings and/or from histopathological examination of specimen. In the group that did not undergo surgery, the standard of reference was the clinical consensus based on follow-up over a period ranging from six to eight weeks.

\section{Statistical analysis}

All the data was processed SPSS statistical packages. Sensitivity, specificity, positive predictive value, negative predictive value and accuracy was calculated for each of the findings and also the overall diagnosis in CT and USG.

\section{RESULTS}

Totally, 61 patients (40 males and 21 females) aged 7 - 68 years (mean age 29.8 years) clinically suspected with acute appendicitis underwent USG and CT. Out of these patients, 28 patients ( 22 males and 6 females) had appendicectomies25 as emergency and 3 as interval appendicectomy (one due to appendicular phlegmon and 2 due to clinical improvement) and appendicitis was confirmed by microscopic examination of the surgical specimen. Appendicectomy was not performed in 33 patients. Among these patients, 14 had an alternate diagnosis ( 8 males and 6 females) (Table 1). The remaining 19 patients (10 males and 9 females) were considered to have nonspecific abdominal pain as their symptoms could not be elucidated with any diagnostic modality and resolved without any specific treatment. They were followed up for 6-8 weeks. All of them had complete relief of symptoms within 36-48 hours and none had recurrent pain during the follow-up period. These patients could be classified as not having appendicitis based on the clinical profile.

\section{Ultrasound}

\begin{tabular}{ccc}
\multicolumn{3}{c}{ Table 1: Summary of number of cases confirmed } \\
\hline & N & $\%$ \\
\hline Confirmation of appendicitis insuspected cases & $28 / 61$ & $45.9 \%$ \\
No appendicitis in clinicallysuspected cases & $33 / 61$ & $54.1 \%$ \\
Alternative diagnosis established & $14 / 61$ & $21.3 \%$ \\
\hline
\end{tabular}

Ultrasound was positive for appendicitis in 27 cases which constituted of;

- Acute appendicitis - 20 (17 confirmed, 2 had appendicular mass, 1 false positive)

- Acute appendicitis with appendicular phlegmon -3 (2 confirmed and 1 false positive)

- Appendicular abscess - 4 ( 3 confirmed, 1 false positive)

There were 25 cases confirmed by surgery, identified as true positive cases. Two cases reported as acute appendicitis had appendicitis with early mass formation during surgery. In 1 case reported as appendicitis, surgery was not performed and the patients improved clinically. This was taken as false positive. In another case which was reported as appendicitis with appendicular phlegmon, CT was normal and the patient became asymptomatic in the follow-up period. This was also taken as false positive. In one case, dermoid ovary was falsely reported as appendicular abscess. This diagnosis was confirmed by CT. Of the 34 cases in which USG was negative for appendicitis, 12 cases had alternative diagnosis. The remaining 22 cases were reported as normal, of which 4 had appendicitis, and 1 case had alternate diagnosis (epiploicappendagitis)(Table2).USG failed to detect appendicitis in 4 surgically proven cases of appendicitis. These were false negative cases.

Table 2: USG diagnosis vs Final diagnosis

\begin{tabular}{ccccccc}
\hline Final diagnosis & Normal & Appendicitis & $\begin{array}{c}\text { Alternate } \\
\text { diagnosis }\end{array}$ & Total & Percentage \\
\cline { 1 - 1 } USG diagnosis & 17 & 4 & 1 & 22 & $36.06 \%$ \\
Normal & 2 & 24 & 1 & 27 & $44.26 \%$ \\
Appendicitis & 0 & 0 & 12 & 12 & $19.67 \%$ \\
\hline
\end{tabular}

Sonography has a sensitivity of $85.71 \%$, specificity of $90.91 \%$ and accuracy of $88.52 \%$ for the diagnosis of acute appendicitis.

CT Diagnosis

CT was positive for appendicitis in 29 cases which constituted of

- Acute appendicitis- 22 (21 confirmed, 1 false positive)

- Acute appendicitis with appendicular mass -4 (confirmed)

- Appendicular abscess - 3 (confirmed) 
There were 28 cases confirmed by surgery, identified as true positive case. CT detected all case of acute appendicitis. One case reported as appendicitis by CT was diagnosed as non-specific pain as the patient's symptoms resolved during the follow-up and never recurred. This was considered as false positive. All 4 appendicular mass and all 3 appendicular abscesses were correctly diagnosed by CT. Of the 32 cases in whom CT was negative for appendicitis, 10 cases had alternate diagnosis by CT. The remaining 22 cases were reported as normal of which 4 had alternate diagnosis ( 2 pelvic inflammatory disease, 1 inguinal hernia, and 1 duodenal perforation peritonitis) (Table 3).

\begin{tabular}{ccccccc}
\multicolumn{7}{c}{ Table 3: CT versus Final diagnosis } \\
\cline { 1 - 4 } Final diagnosis & Normal & Appendicitis & $\begin{array}{c}\text { Alternate } \\
\text { diagnosis }\end{array}$ & Total & Percentage \\
\cline { 1 - 4 } CT diagnosis & & 0 & 4 & 22 & 36.06 \\
\cline { 1 - 2 } $\begin{array}{c}\text { Normal } \\
\text { Appendicitis }\end{array}$ & 18 & 28 & 0 & 29 & 47.54 \\
$\begin{array}{c}\text { Alternate } \\
\text { diagnosis }\end{array}$ & 0 & 0 & 10 & 10 & 16.39 \\
Total & 19 & 28 & 14 & 61 & \\
\hline
\end{tabular}

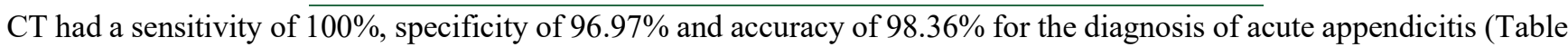
4). There was discordance between CT and USG in the diagnosis of appendicitis in 6 cases. CT diagnosis was correct in all 6 cases ( 2 false positive in USG were correctly reported as no appendicitis by CT, and 4 cases missed by USG were correctly diagnosed as appendicitis by $\mathrm{CT}$ ).

Table 4: Performances of US and CT in the overall diagnosis of acute appendicitis

\begin{tabular}{cccccc}
\hline Modality & Sensitivity & Specificity & PPV & NPV & Accuracy \\
\hline Ultrasound & $85.71(24 / 28)$ & $90.91(30 / 33)$ & $88.89(24 / 27)$ & $88.23(30 / 34)$ & 88.52 \\
CT & $100(28 / 28)$ & $96.96(32 / 33)$ & $96.55(28 / 29)$ & $100(32 / 32)$ & 98.36 \\
\hline
\end{tabular}

Alternate diagnosis

Fourteen patients had alternative diagnosis, of which sonography identified ${ }^{11}$ correctly.One perforation peritonitis was identified as minimal ascites. One epiploic appendagitis was reported as normal. One dermoid ovary was reported as appendicular abscess. CT identified 10 correctly. One D1 perforation peritonitis was missed. Two case of PID was reported as normal. One inguinal hernia was reported as normal. The commonest detectable condition mimicking appendicitis was hydronephrosis ( 3 cases - 25\%). However, the ureteric calculus was identified in only two cases by ultrasound. There was discordance between CT and USG findings in $11(18.03 \%)$ of the 61 patients. The CT diagnosis was correct in $7(63.63 \%)$ patients ( 4 false negative for appendicitis in USG was true positive in CT, 1 false positive for appendicitis by USG was true negative by CT, 1 dermoid ovary was reported as appendicular abscess by USG, 1 epiploicappendag it is was reported as normal by USG).The USG diagnosis was correct in 3 (27.27\%) patients (2 Pelvic inflammatory disease reported as normal by CT, 1 Inguinal hernia was reported as normal by CT). Both CT and USG missed a D1 perforation peritonitis.

Other findings

Visualization of appendix

In USG, appendix was visualised in ${ }^{19}$ case of appendicitis, and 6 cases with no appendicitis ( 2 had alternate diagnosis). Appendix was not visualised in ${ }^{9}$ cases of appendicitis, of which 3 cases were appendicular abscess, ${ }^{2}$ were appendicular mass and 4 had appendicitis. These 4 patients were reported as normal (false positive). In CT, appendix was visualised in 22 cases of appendicitis and 15 cases with no appendicitis of which 5 had alternate diagnosis. Appendix was not visualised in 6 cases of appendicitis of which 3 cases had appendicular abscess and 3 had appendicular mass. Inflamed appendix was visualised in $78.6 \%$ cases of appendicitis by CT compared to $67.9 \%$ by USG. In the 19 patients with nonspecific pain normal appendix was visualised in $52.63 \%$ (10) of cases by CT compared to only $21.05 \%$ (4) by USG.CT was able to exclude appendicitis by demonstrating normal appendix in more cases.

Outer diameter of appendix

The outer diameter of appendix in appendicitis ranged from $8.2 \mathrm{~mm}$ to $16.5 \mathrm{~mm}$ in USG, and from $6.2 \mathrm{~mm}$ to $17.5 \mathrm{~mm}$ in CT. the mean diameter of appendix in appendicitis by USG is $11.01 \mathrm{~mm}$ and by CT is $10.62 \mathrm{~mm}$. For US and CT, the difference in mean diameters in patients with and patients without a definite diagnosis of acute appendicitis was statistically 
significant $(P<0.001$ for both US and CT).

Lack of compressibility

Lack of compressibility was seen in 20 cases out of the 25 cases in which appendix was visualized by USG. Of these 19 cases had appendicitis. 5 cases which were compressible did not have appendicitis. This finding has a positive predictive value of $95 \%$ and negative predictive value of $100 \%$.

Lumen

None of the cases with gas in the appendiceal lumen demonstrated either by CT or USG had appendicitis. This parameter can be used to exclude appendicitis. All the 19 cases of appendicitis in which appendix were visualized by USG and the 22 cases of appendicitis in which appendix were visualized by CT had fluid in the lumen. This sign has high sensitivity in diagnosing appendicitis.

Appendicolith

Appendicolith was identified in 3 cases of appendicitis by USG ( 2 in base, 1 extra luminal).The case with extra luminal appendicolith had appendicular abscess. By CT, appendicolith was identified in 8 cases of appendicitis ( 4 in the base, 2 extra luminal, and 2 multiple with in the lumen). The 2 cases with extra luminal appendicolith had appendicular abscess. Thus the presence of appendicolith has $100 \%$ positive predictive value for diagnosis appendicitis. Also the identification of extra luminal appendicolith is a sign of perforation. CT was more sensitive in identification of appendicolith. Pericaecal fluid was present in 8 cases during sonography ( 3 had appendicitis, 3 had appendicular abscess and 2 had appendicitis with mass during surgery).

Caecal wall thickening

Caecal wall thickening was present only in $18(64.3 \%)$ cases with appendicitis in CT.

Appendicular phlegmon

There were ${ }^{4}$ cases of appendicular phlegmon. Three cases underwent emergency appendicectomies. They had early mass formation. One patient underwent interval appendicectomy. All 4 had histologically proven appendicitis.CT reported all 4 cases correctly. USG diagnosis was correct in 2 cases. However, the other 2 cases of appendicular phlegmon were reported as appendicitis. One case was falsely reported as appendicular phlegmon by USG; CT was normal in this case.

Appendicular abscess

Three cases had appendicular abscess secondary to appendicular perforation, confirmed by surgery. Both CT and USG diagnosed all the 3 cases as appendicular abscess. However, 1 case of dermoid right ovary was reported as appendicular abscess by USG (false positive).

Others

Local ileus, lymphadenopathy and probe tenderness were seen in 15,(57.57\%) 5,(17.85\%) and $25(89.28 \%)$ cases of appendicitis, respectively. Sensitivity and specificity of USG and CT for other parameters in the diagnosis of appendicitis is given in table 5 .

Table 5: Diagnostic Performances of US and CT findings

\begin{tabular}{cccccc}
\hline Findings & Sensitivity & $\begin{array}{c}\text { Specificity } \\
\text { Ultrasound }\end{array}$ & PPV & NPV & Accuracy \\
\hline Outer dia $\geq 6 \mathrm{~mm}$ & $100(19 / 19)$ & $83.33(5 / 6)$ & $95(19 / 20)$ & $100(5 / 5)$ & 96 \\
Lack of compressibility & $100(19 / 19)$ & $83.33(5 / 6)$ & $95(19 / 20)$ & $100(5 / 5)$ & 96 \\
Fluid filled lumen & $100(19 / 19)$ & $50(3 / 6)$ & $86.36(19 / 22)$ & $100(3 / 3)$ & 88 \\
Appendicolith & $10.72(3 / 28)$ & $100(33 / 33)$ & $100(3 / 3)$ & $56.89(33 / 58)$ & 59.01 \\
Hyperechoic periappendiceal & $78.57(22 / 28)$ & $93.33(31 / 33)$ & $91.67(22 / 24)$ & $83.78(31 / 37)$ & 86.88 \\
Tissue & $28.57(8 / 28)$ & $100(33 / 33)$ & $100(8 / 8)$ & $62.26(33 / 53)$ & 67.21 \\
Pericecal fluid & $7.14(2 / 28)$ & $96.97(32 / 33)$ & $66.67(2 / 3)$ & $55.17(32 / 58)$ & 55.73 \\
Phlegmon & $10.71(3 / 28)$ & $96.96(32 / 33)$ & $75(3 / 4)$ & $56.14(32 / 57)$ & 57.37 \\
Abscess & $53.57(15 / 28)$ & $75.75(25 / 33)$ & $65.21(15 / 23)$ & $65.78(25 / 36)$ & 65.57 \\
Local ileus & $17.85(5 / 28)$ & $96.96(32 / 33)$ & $83.33(5 / 6)$ & $58.18(32 / 55)$ & 60.65 \\
Lymphadenopathy & $89.28(25 / 28)$ & $54.54(18 / 33)$ & $62.5(25 / 40)$ & $85.71(18 / 21)$ & 70.49 \\
Probe tenderness & $100(22 / 22)$ & $93.33(14 / 15)$ & $95.65(22 / 23)$ & $100(14 / 14)$ & 97.29 \\
$\quad 100(22 / 22)$ & $73.33(11 / 15)$ & $84.61(22 / 26)$ & $100(11 / 11)$ & 89.18 \\
\hline Outer dia>=6mm & & &
\end{tabular}




\begin{tabular}{cccccc}
\hline Appendicolith & $28.57(8 / 28)$ & $100(33 / 33)$ & $100(8 / 8)$ & $62.26(33 / 53)$ & 67.21 \\
Periappendiceal fat stranding & $96.42(27 / 28)$ & $93.93(31 / 33)$ & $93.10(27 / 29)$ & $96.87(31 / 32)$ & 95.08 \\
Caecal wall thickening & $64.28(18 / 28)$ & $96.96(32 / 33)$ & $94.73(18 / 19)$ & $76.19(32 / 42)$ & 81.96 \\
Phlegmon & $14.28(4 / 28)$ & $100(33 / 33)$ & $100(4 / 4)$ & $57.89(33 / 57)$ & 60.65 \\
Abscess & $10.71(3 / 28)$ & $100(33 / 33)$ & $100(3 / 3)$ & $57.89(33 / 57)$ & 59.01 \\
\hline
\end{tabular}

\section{DISCUSSION}

The usefulness of sonography and $\mathrm{CT}$ in the diagnosis of appendicitis has been reported extensively in the literature. As the literature related to surgery report an average rate of $50 \%$ negative findings at appendectomy, ${ }^{1-3}$ imaging techniques are useful to prevent unnecessary appendectomies and to avoid costly hospital admissions. Furthermore, imaging can expedite the diagnosis of appendicitis, minimizing surgical delays and the subsequent risk of appendiceal perforation. In this study, 61 patients clinically suspected of appendicitis underwent graded compression sonography and unenhanced focused CT. Of these, $28(45.9 \%)$ had acute appendicitis and 33 (54.1\%) did not have appendicitis. USG diagnosed 24 cases of appendicitis and 30 cases without appendicitis correctly. There were 3 false positive and 4 false negative cases. CT diagnosed all 28 cases of appendicitis and 32 cases without appendicitis correctly. There was one false positive case. In the present study, the graded compression sonography has sensitivity $(85.7 \%)$, specificity $(90.9 \%)$, positive predictive value $(88.9 \%)$, negative predictive value $(88.2 \%)$ and accuracy $(88.5 \%)$ whereas, unenhanced focused CT has sensitivity $(100 \%)$, specificity (96.9\%),positive predictive value $(96.6 \%)$, negative predictive value (100\%) and accuracy (98.4\%). The diagnostic performance of graded compression sonography and unenhanced focused CT were comparable to published literature. ${ }^{4-10}$ The study by Ege G et al ${ }^{10}$ who used similar CT protocol for imaging patients reported a sensitivity of $96 \%$, specificity of $98 \%$, positive predictive value of $97 \%$, negative predictive value of $98 \%$ and accuracy of $97 \%$. This is comparable to the present study. In this study, the unenhanced focused CT showed better diagnostic performances compared to graded compression sonography for the diagnosis of appendicitis but there was no significant difference in sensitivity, specificity, predictive values or accuracy between these imaging techniques. The results of present study are similar to that of Poortman et al, ${ }^{11}$ who, when comparing the graded compression technique of US with focused unenhanced $\mathrm{CT}$, reported no significant difference in sensitivity, specificity, or accuracy between these imaging techniques. In this study, discordance between $\mathrm{CT}$ and sonographic findings occurred in $11(16 \%)$ of 61 cases undergoing both examinations. The diagnosis by $\mathrm{CT}$ was correct in 7 $(63.6 \%)$ of the cases, whereas the sonographic diagnosis was correct in only $3(27.3 \%)$ cases. Sonography was slightly better when compared to the study by Sivit CJ et $a l^{12}$ who got correct diagnosis by CT in $17 / 20(85 \%)$ of the cases, compared to the sonographic diagnosis which was correct in only $3 / 20(15 \%)$ cases. This may be because sonography was better in diagnosing pelvic inflammatory disease in females compared to unenhanced $\mathrm{CT}$ in the present study. However, CT was correct in the diagnosis of appendicitis in all $6(100 \%)$ cases in which the CT and USG diagnosis differed. This is comparable to the study by Sivit CJ et al ${ }^{12}$ in which CT was correct in $98 \%$ cases. Thus, CT is more likely to be correct when there is a discordant diagnosis. An important issue to be addressed while evaluating the positive impact of an imaging examination on the assessment of patients with suspected appendicitis is the value of normal findings on an examination using a modality. The higher the sensitivity of the imaging examination, the lower the number of falsenegative examinations, and, consequently, more trust can be placed on normal findings from that examination by caregivers. Because of the significantly higher sensitivity of CT $(100 \%)$ when compared with sonography $(85.7 \%)$ for the diagnosis of appendicitis, a negative CT examination in stills greater diagnostic confidence for the exclusion of appendicitis than do negative sonographic findings. Thus, use of CT can avoid unnecessary appendicectomy. Several other factors emphasize the value of CT over USG in patients with suspected acute appendicitis. Unenhanced focused $\mathrm{CT}$ was able to visualize $37(60.7 \%)$ of appendices compared to $25(41 \%)$ by USG. The visualization of appendix in appendicitis was also higher with $\mathrm{CT}$, with $\mathrm{CT}$ being able to show inflamed appendix in $22(78.6 \%)$ of cases compared to $19(67.9 \%)$ by USG. Visualization of normal appendix in patients who do not have appendicitis can exclude appendicitis. This is particularly important in patients who have normal findings in the examination and do not have an alternative diagnosis as well. In this group, normal appendices was visualized in $10(52.63 \%)$ of cases by CT compared to 4 $(21 \%)$ by USG. In the study by Balthazar et al,,${ }^{13}$ the normal appendix, when present, was noted $48 \%$ of the time by CT and only $4 \%$ of the time by USG.

The ability to exclude appendicitis, however, requires visualization of the entire normal appendix, which is an uncommon occurrence by sonography. In his initial study, Puylaert $^{8}$ did not visualize normal appendices. Recently several groups reported a higher rate of normal appendices in up to $50 \%$ of cases. ${ }^{14}$ However, many studies were 
performed in asymptomatic patients which could explain their higher detection rates. In the present study, normal appendices was visualised in $6(18.18 \%)$ of cases by sonography. Outer diameter $\geq 6 \mathrm{~mm}$ as the criteria for diagnosing appendicitis has a sensitivity of $100 \%$ both in CT and USG. However, 1 case with no appendicitis had a diameter of $>6 \mathrm{~mm}(6.2 \mathrm{~mm}$ in USG and $6.4 \mathrm{~mm}$ in CT). This has reduced the specificity of the parameter. This result stressed the fact that isolated finding of an appendix with a diameter exceeding $6 \mathrm{~mm}$ is an insufficient basis for a diagnosis of acute appendicitis. This finding when combined with findings of inflammatory changes involving the thickened appendix (i.e., streaking and poorly defined increased attenuation in the periappendiceal fat in CT or hyperechoic periappendiceal fat in USG) can increase the specificity. CT is better in showing the periappendiceal inflammatory changes. According to Malone $\mathrm{AJ}^{15}$ the identification of inflammatory changes in the pericecal and periappendiceal fat are the most important findings when the diagnosis of acute appendicitis is done by unenhanced CT. Balthazar et $a l^{16}$ found that lack of visualization of an abnormal appendix in contrast- enhanced CT scans, even in the presence of obvious inflammatory changes in the right lower quadrant, is a nonspecific finding and is an insufficient basis for the diagnosis of acute appendicitis. In the present study, periappendiceal inflammatory changes were taken as supportive evidence of appendicitis. The periappendiceal inflammation seen as periappendiceal/ pericecalhyperechoic fat was seen in $22(78.57 \%)$ cases of appendicitis in USG. Similarly, periappendiceal/ pericecal fat stranding was seen in $27(96.42 \%)$ cases of appendicitis. This again highlights the fact that periappendiceal inflammation was better shown in CT. CT identified $8(28.6 \%)$ cases of appendicoliths compared to 3 $(10.7 \%)$ by USG. Two cases of appendicular abscess had appendicolith in extraluminal location. The presence of appendicolith demonstrated by CT or USG had a $100 \%$ specificity and positive predictive value for the diagnosis of appendicitis although the sensitivity was less. In the present study, there was no significant difference between CT and USG in showing the abscess (14.2\% vs $10.7 \%)$. However, CT was more sensitive in identifying phlegmon (14.3\% vs 7.14\%). Based on the above findings in this prospective comparative study, CT was superior to US in evaluating patients suspected of having acute appendicitis.

\section{CONCLUSION}

This study shows that both unenhanced focused CT and sonography are accurate imaging modalities in patients with suspected appendicitis. The choice of type of study to perform is likely to depend on the available resources and personnel at various institutions and the clinical features. However, CT was found to be superior to US in evaluating patients suspected of having acute appendicitis.

\section{REFERENCES}

1. Birnbaum B, Wilson S. Appendicitis at the millennium. Radiology 2000; 215:337-348

2. Pieper R, Kager L, Nasman P. Acute appendicitis: a clinical study of 1018 cases of emergency appendectomy. ActaChirScand 1982; 148:51-62.

3. Borgstein PJ, Eijsbouts QAJ, De Jong D, Gordijn RV, Cuesta MA. Acute appendicitis: a clear-cut case in men, a guessing game in young women - a prospective study on the role of laparoscopy. SurgEndosc 1997;11:923-927.

4. Birnbaum BA, Jeffrey RB Jr. CT and sonographic evaluation of acute right lower abdominal pain. AJR Am J Roentgenol 1998;170(2):361-71.

5. Abu-Yousef MM, Bleicher JJ, Maher JW, Urdaneta LF, Franken EA Jr, Metcalf AM. High-resolution sonography of acute appendicitis. AJR Am J Roentgenol1987;149:53-8.

6. Puylaert JB. US evaluation using graded compression. Radiology 1986;158:355-60.

7. Rao PM, Rhea JT, Novelline RA, Mostafavi AA, Lawrason $\mathrm{JN}$, McCabe CJ. Helical CT combinedwith contrast material administered only through the colon for imaging of suspected appendicitis.AJR Am J Roentgenol 1997;169:1275-80.

8. Rao PM, Rhea JT, Novelline RA, et al. Helical CT technique for the diagnosis of appendicitis:prospective evaluation of a focused appendix CT examination. Radiology 1997; 202:13944.

9. Fitz RH. Perforating inflammation of the vermiform appendix; with special reference to its earlydiagnosis and treatment. Am J Med Sci 1886;92:321-46.

10. Hermans JJ, Hermans AL, Risseeuw GA, Verhaar JC, Meradji M. Appendicitis caused by carcinoid tumor. Radiology 1993;188:71-2.

11. Zebrowska G, Walsh NM. Human immunodeficiency virusrelated Kaposi's sarcoma of the appendix and acute appendicitis. Report of a case and review of the literature. Arch Pathol Lab Med 1991;115:1157-60.

12. Wilcox RT, Traverso LW. Have the evaluation and treatment of acute appendicitis changed with new technology? SurgClin N Am 1997;77:1355-70.

13. Guidry SP, Poole GV. The anatomy of appendicitis. Am Surg 1994; 60:68-71.

14. Yabunaka K, Katsuda T, Sanada S, Fukutomi T. Sonographic appearance of the normal appendix in adults. J Ultrasound Med 2007;26:37-43

15. Malone AJ, Wolf CR, Malmed AS, Melliere BF. Diagnosis of acute appendicitis: value of unenhanced CT. AJR Am J Roentgenol 1993;160:763-6.

16. Buschard K, Kjaeldfaard A. Investigation and analysis of the position, fixation, length and embryology of the vermiform appendix. ActaChirScand 1973;139:293-8.

\section{Source of Support: None Declared Conflict of Interest: None Declared}

